\title{
An Efficient 2-D DOA Estimation for a Cylindrical Conformal Array with Unknown Mutual Coupling
}

\author{
Chao Liu (iD) and Shunian Yin \\ Department of Communication Engineering, Hefei University of Technology, Hefei, Anhui 230601, China \\ Correspondence should be addressed to Chao Liu; disneyl@hfut.edu.cn
}

Received 2 February 2018; Revised 27 June 2018; Accepted 5 July 2018; Published 4 October 2018

Academic Editor: Renato Cicchetti

Copyright (c) 2018 Chao Liu and Shunian Yin. This is an open access article distributed under the Creative Commons Attribution License, which permits unrestricted use, distribution, and reproduction in any medium, provided the original work is properly cited.

\begin{abstract}
The limited space of a conformal array may lead to a serious mutual coupling effect, which will significantly affect the performance of direction of arrival (DOA) estimation algorithms. In this paper, an efficient 2-D direction finding method is developed in the presence of unknown mutual coupling for the uniform cylindrical conformal array (CCA). To avoid the time-consuming twodimensional spectral peak searching, the 2-D DOA estimation is decoupled and divided into two 1-D DOA estimations. Elevation is first estimated based on a subarray estimation of signal parameters via rotation invariant technique (ESPRIT), and then azimuth is estimated based on the rank reduction (RARE) method by using the elevation estimation result. Consequently, the mutual coupling coefficients can be estimated after getting the DOA estimates. The proposed method can well calibrate the mutual coupling effect of a cylindrical array with a low computational complexity. The final simulation results corroborate our analysis.
\end{abstract}

\section{Introduction}

Direction of arrival (DOA) estimation is a major research direction in array signal processing area. It has broad application prospects in many occasions, and lots of algorithms have been developed in the past decades. However, most of them are only applicable to planar arrays. Conformal array has a lot of advantages such as good aerodynamic performance, wide surveillance area, and low radar cross-section. Therefore, it has drawn extensive attention in recent years [1]. Compared with the planar array, the curved structure of a conformal array brings new challenges to the problem of DOA estimation. Moreover, due to the limited arrange space of a conformal array, the effect of mutual coupling may become more obvious and consequently degrade the performance of most existing DOA estimation algorithms [2].

Researchers have been focused on this problem, and a large number of algorithms have been proposed to calibrate the imperfect manifold caused by mutual coupling. In [3], mutual coupling effect was calibrated as well as sensor location error and phase/gain uncertainty by an iterative process. In [4], a unified framework was formulated to express the typical array perturbations and a sparse Bayesian method was proposed for array calibration and DOA estimation. However, the convergence of these iterative methods was not theoretical guaranteed and the associated multidimensional search and iterations were time consuming. By taking advantages of the special structure of uniform linear array (ULA) and uniform rectangular array (URA), auxiliary elements were demonstrated to be effective for the autocalibration of mutual coupling [5-7]. This kind of method was further extended to the situation of non-Gaussian noise [8] and coherent signal condition [9] by using fourth-order cumulants (FOC) and spatial smoothing technique, respectively. These auxiliary algorithms can accurately estimate DOAs without any calibration source or iteration procedure. However, there is an aperture loss for these methods and they can only be applied to uniform arrays.

The rank reduction (RARE) algorithm is another class of methods for DOA estimation with unknown mutual coupling [10-13]. Through matrix transformation, the coupling effect is eliminated and a rank reduction matrix is defined to estimate the DOAs. In [12], an iterative process was introduced to further improve the DOA estimation 


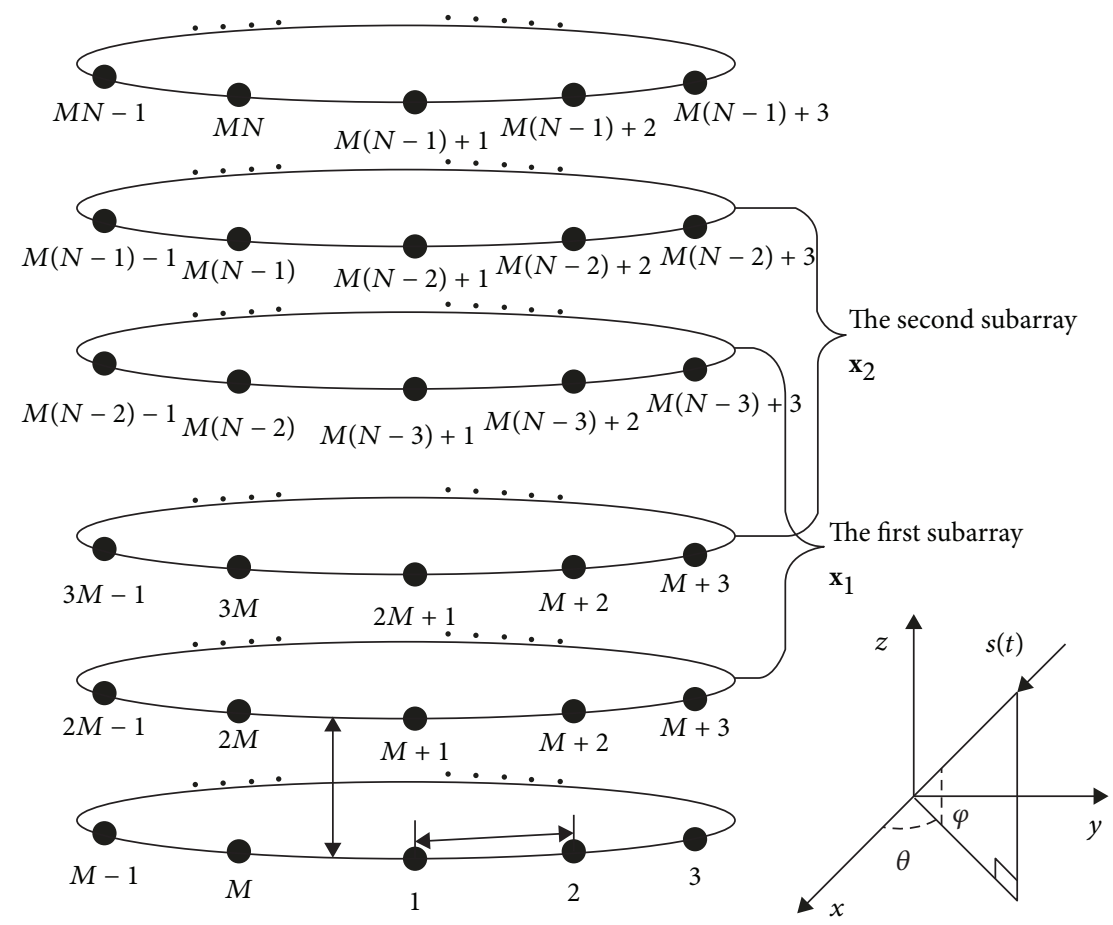

FIgURE 1: Geometry and element numbering of CCA.

accuracy. In [13], a recursive RARE method for DOA estimation in the presence of unknown mutual coupling was presented. It recursively built up RARE matrices by the angle estimation to overcome the problem of nonunique coupling coefficient estimation and gave a better DOA estimation performance than the original RARE method. Generally, these methods use all the array elements rather than the middle subarray to avoid the array aperture loss and have a better performance than the auxiliary methods, especially for small arrays. Other methods are also proposed to calibrate the coupling effect. In [14], mutual coupling matrix (MCM) was reconstructed to calibrate the mutual coupling effect, in which pattern matrix for the embedded elements and for the isolated elements needed to be known in prior. By using the special structure of the uniform linear array, a Toeplitz matrix was reconstructed with the receiving data to replace the covariance matrix [15]. Based on that, subspace-based methods can be used without any compensation.

However, most of the above methods can only be applied to the linear or planar array. Mutual coupling calibration for a conformal array is more complicated, and few researches have been involved in this problem so far. In [16], the RARE algorithm was adopted to estimate DOAs for the conformal array. In [17], a tensor 2-D DOA estimation was proposed for cylindrical conformal array (CCA) under unknown mutual coupling. However, only the mutual coupling between elements in one ULA is considered, which simplifies the problem. Moreover, both of the above methods involved spectrum searching and lots of matrix computation and the amount of calculation will dramatically increase in a 2-D case.
In this paper, an efficient decoupling method is proposed to estimate the 2-D DOAs and the mutual coupling coefficients for CCA. The elevation is first estimated based on a subarray ESPRIT method. By using the elevation estimate, azimuth can be further estimated based on a 1-D RARE method. After that, mutual coupling coefficients can subsequently be solved. The 2-D DOA estimation is decoupled and divided into two individual 1-D DOA estimations, which can significantly reduce the computational complexity. Numerical experiments are provided to demonstrate the effectiveness of the proposed method.

\section{Signal Model}

Consider a uniform CCA with the size of $M \times N$, in which each layer is an $N$ element uniform circular array (UCA) and each column is an $M$ element ULA. The array geometry, coordinate system, and element numbering are shown in Figure 1. The space between neighbouring sensors in UCA is $d_{x y}$ and that of ULA is $d_{z}$. Assume that there are $K$ signals, $s_{i}(t), i=1,2, \ldots, K$, arriving at the array from directions $\left(\theta_{1}, \varphi_{1}\right),\left(\theta_{2}, \varphi_{2}\right), \ldots,\left(\theta_{K}, \varphi_{K}\right)$, respectively. The symbols $\theta_{i}$ and $\varphi_{i}$ denote the azimuth and elevation of the $i$ th signal. Assuming that the MCM of CCA is C, then the array output vector $\mathbf{x}(t)$ can be written as

$$
\mathbf{x}(t)=\mathbf{C A s}(t)+\mathbf{n}(t)
$$

The $M N \times 1$ output signal vector $\mathbf{x}(t)$, the $K \times 1$ source signal vector $\mathbf{s}(t)$, and the $M N \times 1$ noise vector $\mathbf{n}(t)$ in (1) are defined as follows. 


$$
\begin{aligned}
\mathbf{x}(t) & =\left[x_{1}(t), \ldots, x_{M}(t), \ldots, x_{2 M}(t), \ldots, x_{M(N-1)+1}(t), \ldots, x_{M N}(t)\right]^{T}, \\
\mathbf{s}(t) & =\left[s_{1}(t), \ldots, s_{K}(t)\right]^{T}, \\
\mathbf{n}(t) & =\left[n_{1}(t), \ldots, n_{M}(t), \ldots, n_{2 M}(t), \ldots, n_{M(N-1)+1}(t), \ldots, n_{M N}(t)\right]^{T} .
\end{aligned}
$$

The array manifold matrix $\mathbf{A}$ is defined as

$$
\begin{aligned}
& \mathbf{A}=\left[\mathbf{a}\left(\theta_{1}, \varphi_{1}\right), \mathbf{a}\left(\theta_{2}, \varphi_{2}\right), \ldots, \mathbf{a}\left(\theta_{K}, \varphi_{K}\right)\right], \\
& \mathbf{a}\left(\theta_{i}, \varphi_{i}\right)= {\left[g_{1}\left(\theta_{i}, \varphi_{i}\right) \exp \left(j k_{0} \mathbf{r}_{1}^{T} \mathbf{v}_{i}\right), g_{2}\left(\theta_{i}, \varphi_{i}\right)\right.} \\
&\left.\cdot \exp \left(j k_{0} \mathbf{r}_{2}^{T} \mathbf{v}_{i}\right), \ldots, g_{M N}\left(\theta_{i}, \varphi_{i}\right) \exp \left(j k_{0} \mathbf{r}_{M N}^{T} \mathbf{v}_{i}\right)\right]^{T}, \\
& i=1, \ldots, K,
\end{aligned}
$$

where $k_{0}=2 \pi / \lambda$ is the wave number, $\mathbf{r}_{j}=\left[x_{j}, y_{j}, z_{j}\right]^{T}$ is the coordinate vector of the $j$ th element, $\mathbf{v}_{i}=\left[\cos \theta_{i} \cos \varphi_{i}\right.$, $\left.\sin \theta_{i} \cos \varphi_{i}, \sin \varphi_{i}\right]^{T}$ is the unit vector of direction $\left(\theta_{i}, \varphi_{i}\right)$, and $g_{j}(\theta, \varphi)$ is the element pattern of the $j$ th element defined in the global coordinate system.

It is found that mutual coupling effect decreases along with the increase of space between elements [18]. Without loss of generality, assume that each sensor only suffers from the mutual coupling effect of its eight neighbouring elements, and the mutual coupling effect model is shown in Figure 2. The coefficients are assumed to satisfy the relationship $\left|c_{x z}\right|<\left|c_{x}\right|,\left|c_{x z}\right|<\left|c_{z}\right|$. The whole CCA can be seen as a compound ULA composed of several identical UCAs. Then the MCM $\mathbf{C}$ of the whole array can be expressed as follows.

$$
\mathbf{C}=\left[\begin{array}{ccccc}
\mathrm{C}_{1} & \mathrm{C}_{2} & \mathbf{0} & \ldots & \mathbf{0} \\
\mathrm{C}_{2} & \mathrm{C}_{1} & \mathrm{C}_{2} & \ldots & \mathbf{0} \\
\vdots & \ddots & \ddots & \ddots & \vdots \\
\mathbf{0} & \ldots & \mathrm{C}_{2} & \mathrm{C}_{1} & \mathrm{C}_{2} \\
\mathbf{0} & \ldots & \mathbf{0} & \mathrm{C}_{2} & \mathrm{C}_{1}
\end{array}\right]_{M N \times M N}
$$

where $\mathrm{C}_{1}$ is the MCM of each UCA subarray and $\mathbf{C}_{2}$ is the MCM between two neighbouring UCAs. Due to the uniformly spaced structure, they are symmetric Toeplitz matrices [18], and can be expressed as

$$
\begin{aligned}
& \mathbf{C}_{1}=\operatorname{Toeplitz}\left\{\left[1, c_{x}, 0, \ldots, 0, c_{x}\right]_{1 \times M}\right\}, \\
& \mathbf{C}_{2}=\operatorname{Toeplitz}\left\{\left[c_{z}, c_{x z}, 0, \ldots, 0, c_{x z}\right]_{1 \times M}\right\} .
\end{aligned}
$$

\section{DOA Estimation for CCA with Unknown Mutual Coupling}

In this section, dimensionality reduction is first carried out to decrease the computational complexity. The 2-D DOA estimation for CCA is divided into two 1-D DOA processes,

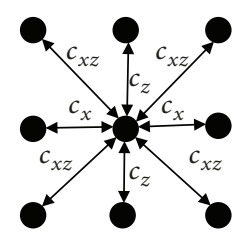

FIGURE 2: Mutual coupling effect model.

and then azimuth and elevation of signals are decoupled and estimated, respectively.

3.1. Elevation Estimation. According to the mutual coupling model, the top and bottom layers are set to be auxiliary elements and two subarrays are selected to estimate the elevation of signals. They compose of the same number of layers, which is shown in Figure 1. Hence, the outputs of the two subarrays can be written as

$$
\begin{aligned}
& \mathbf{x}_{1}(t)=\mathbf{P}_{1} \mathbf{x}(t)=\mathbf{P}_{1} \operatorname{CAs}(t)+\mathbf{P}_{1} \mathbf{n}(t), \\
& \mathbf{x}_{2}(t)=\mathbf{P}_{2} \mathbf{x}(t)=\mathbf{P}_{2} \operatorname{CAs}(t)+\mathbf{P}_{2} \mathbf{n}(t),
\end{aligned}
$$

in which $\quad \mathbf{x}_{1}(t)=\left[x_{M+1}(t), \ldots, x_{2 M}(t), \ldots, x_{3 M}(t), \ldots\right.$, $\left.x_{M(N-3)+1}(t), \ldots, x_{M(N-2)}(t)\right]^{T}$ and $\quad \mathbf{x}_{2}(t)=\left[x_{2 M+1}(t), \ldots\right.$, $\left.x_{3 M}(t), \ldots, x_{4 M}(t), \ldots, x_{M(N-2)+1}(t), \ldots, x_{M(N-1)}(t)\right]^{T}$. In (6), $\mathbf{P}_{1}$ and $\mathbf{P}_{2}$ are two screening matrices defined as

$$
\begin{aligned}
& \mathbf{P}_{1}=\left[\begin{array}{ccccccc}
\mathbf{0} & \mathbf{I} & \mathbf{0} & \ldots & \mathbf{0} & \mathbf{0} & \mathbf{0} \\
\mathbf{0} & \mathbf{0} & \mathbf{I} & \ldots & \mathbf{0} & \mathbf{0} & \mathbf{0} \\
\vdots & \vdots & & \ddots & \vdots & \vdots & \vdots \\
\mathbf{0} & \mathbf{0} & \ldots & & \mathbf{I} & \mathbf{0} & \mathbf{0}
\end{array}\right]_{M(N-3) \times M N} \\
& \mathbf{P}_{2}=\left[\begin{array}{ccccccc}
\mathbf{0} & \mathbf{0} & \mathbf{I} & \mathbf{0} & \ldots & \mathbf{0} & \mathbf{0} \\
\mathbf{0} & \mathbf{0} & \mathbf{0} & \mathbf{I} & \ldots & \mathbf{0} & \mathbf{0} \\
\vdots & \vdots & & \ddots & \ddots & \vdots & \vdots \\
\mathbf{0} & \mathbf{0} & \ldots & \ldots & \mathbf{0} & \mathbf{I} & \mathbf{0}
\end{array}\right]_{M(N-3) \times M N}
\end{aligned}
$$

where $\mathbf{0}$ is an $M \times M$ zero matrix and $\mathbf{I}$ is an $M \times M$ identity matrix.

In the presence of mutual coupling, the real steering vectors of the two subarrays are $\mathbf{P}_{1} \mathbf{C a}(\theta, \varphi)$ and $\mathbf{P}_{2} \mathbf{C a}(\theta, \varphi)$. The ideal steering vector of the whole CCA $\mathbf{a}(\theta, \varphi)$ can be rewritten as

$$
\mathbf{a}\left(\theta_{i}, \varphi_{i}\right)=\mathbf{a}_{z}\left(\theta_{i}, \varphi_{i}\right) \otimes \mathbf{a}_{x y}\left(\theta_{i}, \varphi_{i}\right), \quad i=1, \ldots, K
$$

The symbol $\otimes$ denotes the Kronecker product and $\mathbf{a}_{z}\left(\theta_{i}, \varphi_{i}\right)$ and $\mathbf{a}_{x y}\left(\theta_{i}, \varphi_{i}\right)$ denote the steering vectors of the linear and circular subarrays without mutual coupling, respectively. 


$$
\begin{aligned}
& \mathbf{a}\left(\theta_{i}, \varphi_{i}\right)= {\left[g_{1}\left(\theta_{1}, \varphi_{1}\right) \beta_{x y i 1}, \ldots, g_{M}\left(\theta_{i}, \varphi_{i}\right) \beta_{x y i M}\right]^{T}, } \\
& i=1, \ldots, K, \\
& \beta_{x y i j}= \exp \left\{-j k_{0}\left(x_{j} \cos \theta_{i} \cos \varphi_{i}+y_{j} \sin \theta_{i} \cos \varphi_{i}\right)\right\}, \\
& i=1, \ldots, K, j=1, \ldots, M, \\
& \mathbf{a}\left(\theta_{i}, \varphi_{i}\right)=\left[1, \beta_{z i}, \beta_{z i}^{2}, \ldots, \beta_{z i}^{N-1}\right]^{T}, \quad i=1, \ldots, K, \\
& \beta_{z i}=\exp \left\{j k_{0} d_{z} \sin \varphi_{1}\right\}, \quad i=1, \ldots, K .
\end{aligned}
$$

Taking a and $\mathbf{a}_{x y}$ as the shorter terms for $\mathbf{a}(\theta, \varphi)$ and $\mathbf{a}_{x y}(\theta, \varphi)$, respectively, then the steering vectors of the two subarrays can be expressed as follows.

$$
\begin{aligned}
& \mathbf{P}_{1} \mathbf{C a}=\left[\begin{array}{ccccccc}
\mathbf{0} & \mathbf{I} & \mathbf{0} & \ldots & \mathbf{0} & \mathbf{0} & \mathbf{0} \\
\mathbf{0} & \mathbf{0} & \mathbf{I} & \ldots & \mathbf{0} & \mathbf{0} & \mathbf{0} \\
\vdots & \vdots & & \ddots & \vdots & \vdots & \vdots \\
\mathbf{0} & \mathbf{0} & \ldots & & \mathbf{I} & \mathbf{0} & \mathbf{0}
\end{array}\right] \\
& \cdot\left[\begin{array}{ccccc}
\mathbf{C}_{1} & \mathbf{C}_{2} & \mathbf{0} & \ldots & \mathbf{0} \\
\mathbf{C}_{2} & \mathbf{C}_{1} & \mathbf{C}_{2} & \ldots & \mathbf{0} \\
\vdots & \ddots & \ddots & \ddots & \vdots \\
\mathbf{0} & \ldots & \mathbf{C}_{2} & \mathbf{C}_{1} & \mathbf{C}_{2} \\
\mathbf{0} & \ldots & \mathbf{0} & \mathbf{C}_{2} & \mathbf{C}_{1}
\end{array}\right] \cdot\left[\begin{array}{c}
\mathbf{a}_{x y} \\
\beta_{z} \mathbf{a}_{x y} \\
\vdots \\
\beta_{z}^{N-1} \mathbf{a}_{x y}
\end{array}\right] \\
& =\left[\begin{array}{c}
\mathbf{C}_{2} \mathbf{a}_{x y}+\mathbf{C}_{1} \beta_{z} \mathbf{a}_{x y}+\mathbf{C}_{2} \beta_{z}^{2} \mathbf{a}_{x y} \\
\mathbf{C}_{2} \beta_{z} \mathbf{a}_{x y}+\mathbf{C}_{1} \beta_{z}^{2} \mathbf{a}_{x y}+\mathbf{C}_{2} \beta_{z}^{3} \mathbf{a}_{x y} \\
\vdots \\
\mathbf{C}_{2} \beta_{z}^{N-4} \mathbf{a}_{x y}+\mathbf{C}_{1} \beta_{z}^{N-3} \mathbf{a}_{x y}+\mathbf{C}_{2} \beta_{z}^{N-2} \mathbf{a}_{x y}
\end{array}\right],
\end{aligned}
$$

$$
\begin{aligned}
\mathbf{P}_{2} \mathbf{C a}= & {\left[\begin{array}{ccccccc}
\mathbf{0} & \mathbf{0} & \mathbf{I} & \mathbf{0} & \ldots & \mathbf{0} & \mathbf{0} \\
\mathbf{0} & \mathbf{0} & \mathbf{0} & \mathbf{I} & \ldots & \mathbf{0} & \mathbf{0} \\
\vdots & \vdots & & \ddots & \ddots & \vdots & \vdots \\
\mathbf{0} & \mathbf{0} & \ldots & \ldots & \mathbf{0} & \mathbf{I} & \mathbf{0}
\end{array}\right] } \\
& \cdot\left[\begin{array}{ccccc}
\mathbf{C}_{1} & \mathbf{C}_{2} & \mathbf{0} & \ldots & \mathbf{0} \\
\mathbf{C}_{2} & \mathbf{C}_{1} & \mathbf{C}_{2} & \ldots & \mathbf{0} \\
\vdots & \ddots & \ddots & \ddots & \vdots \\
\mathbf{0} & \ldots & \mathbf{C}_{2} & \mathbf{C}_{1} & \mathbf{C}_{2} \\
\mathbf{0} & \ldots & \mathbf{0} & \mathbf{C}_{2} & \mathbf{C}_{1}
\end{array}\right] \cdot\left[\begin{array}{c}
\mathbf{a}_{x y} \\
\beta_{z} \mathbf{a}_{x y} \\
\vdots \\
\beta_{z}^{N-1} \mathbf{a}_{x y}
\end{array}\right]
\end{aligned}
$$

$$
=\left[\begin{array}{c}
\mathbf{C}_{2} \mathbf{a}_{x y}+\mathbf{C}_{1} \beta_{z} \mathbf{a}_{x y}+\mathbf{C}_{2} \beta_{z}^{2} \mathbf{a}_{x y} \\
\mathbf{C}_{2} \beta_{z} \mathbf{a}_{x y}+\mathbf{C}_{1} \beta_{z}^{2} \mathbf{a}_{x y}+\mathbf{C}_{2} \beta_{z}^{3} \mathbf{a}_{x y} \\
\vdots \\
\mathbf{C}_{2} \beta_{z}^{N-4} \mathbf{a}_{x y}+\mathbf{C}_{1} \beta_{z}^{N-3} \mathbf{a}_{x y}+\mathbf{C}_{2} \beta_{z}^{N-2} \mathbf{a}_{x y}
\end{array}\right] \beta_{z} .
$$

By comparing (10) and (11), it can be found that the steering vectors of the two subarrays have similar structures and it can be simplified as the following equation.

$$
\mathbf{P}_{2} \mathbf{C a}=\mathbf{P}_{1} \mathbf{C a} \beta_{z} .
$$

Substitute (12) into (6) and the following equations can be obtained.

$$
\begin{aligned}
& \mathbf{x}_{1}(t)=\mathbf{P}_{1} \mathbf{x}(t)=\mathbf{P}_{1} \mathbf{C A s}(t)+\mathbf{P}_{1} \mathbf{n}(t), \\
& \mathbf{x}_{2}(t)=\mathbf{P}_{2} \mathbf{x}(t)=\mathbf{P}_{2} \operatorname{CAs}(t)+\mathbf{P}_{2} \mathbf{n}(t)=\mathbf{P}_{1} \operatorname{CA} \boldsymbol{\Phi} \mathbf{s}(t)+\mathbf{P}_{2} \mathbf{n}(t),
\end{aligned}
$$

where $\boldsymbol{\Phi}=\operatorname{diag}\left\{\beta_{\mathrm{z} 1}, \ldots, \beta_{\mathrm{zK}}\right\}$. The rotation matrix $\boldsymbol{\Phi}$ only contains the information of signal elevation, which is not affected by mutual coupling. Therefore, the elevation can be estimated by using ESPRIT algorithm. Define a new signal vector $\mathbf{z}(t)=\left[\mathbf{x}_{1}(t)^{T}, \mathbf{x}_{2}(t)^{T}\right]^{T}$. The signal subspaces $\mathbf{U}_{S}$ can be obtained via the eigen decomposition of the covariance matrix of $\mathbf{z}(t)$, and it can subsequently be divided into the up and down halves $\mathbf{U}_{S 1}$ and $\mathbf{U}_{S 2}$. It is easy to derive the following equations.

$$
\begin{aligned}
\mathbf{U}_{S 1} & =\mathbf{U}_{S 2} \psi, \\
\psi & =\mathbf{T}^{-1} \boldsymbol{\Phi} \mathbf{T} .
\end{aligned}
$$

Since $\mathbf{T}$ is a full-rank matrix, $\boldsymbol{\Phi}$ and $\psi$ have the same eigenvalues. Therefore, the elevations can be estimated by the eigenvalues $\phi_{i}, i=1, \ldots, K$ of $\psi$.

$$
\varphi_{i}=\sin ^{-1}\left(\frac{\lambda}{2 \pi d_{z}} \arg \left(\phi_{i}\right)\right), \quad i=1, \ldots, K .
$$

3.2. Azimuth and Mutual Coupling Coefficient Estimation. Based on the above elevation estimation, the azimuth can be estimated by utilizing the output of the whole array. The covariance matrix of $\mathbf{x}(t)$ is defined as

$$
\mathbf{R}=E\left\{\mathbf{x}(t) \mathbf{x}^{H}(t)\right\}=\mathbf{C A R}_{S} \mathbf{A}^{H} \mathbf{C}^{H}+\sigma^{2} \mathbf{I}_{M},
$$

where $\mathbf{R}_{S}=E\left\{\mathbf{s}(t) \mathbf{s}^{H}(t)\right\}$ is the signal covariance matrix. The signal subspace $\mathbf{U}_{s}$ and the noise subspace $\mathbf{U}_{N}$ can be obtained by taking eigen decomposition of the covariance matrix $\mathbf{R}$. The space spans by the steering vectors coincide with the signal subspace and are orthogonal with the noise subspace.

$$
\operatorname{span}\{\mathbf{C A}\}=\operatorname{span}\left\{\mathbf{U}_{s}\right\} \perp \operatorname{span}\left\{\mathbf{U}_{N}\right\} .
$$

Therefore, we have

$$
\mathbf{U}_{N}^{H} \mathbf{C a}\left(\theta_{i}, \varphi_{i}\right)=\mathbf{0}, \quad i=1,2, \ldots K .
$$


As the special structure of the MCM C in (4), the steering vector of the whole array can be rewritten as

$$
\begin{aligned}
& \mathbf{C a}=\left[\begin{array}{ccccc}
\mathrm{C}_{1} & \mathrm{C}_{2} & \mathbf{0} & \ldots & \mathbf{0} \\
\mathrm{C}_{2} & \mathrm{C}_{1} & \mathrm{C}_{2} & \ldots & \mathbf{0} \\
\vdots & \ddots & \ddots & \ddots & \vdots \\
\mathbf{0} & \ldots & \mathrm{C}_{2} & \mathrm{C}_{1} & \mathrm{C}_{2} \\
\mathbf{0} & \ldots & \mathbf{0} & \mathrm{C}_{2} & \mathrm{C}_{1}
\end{array}\right] \\
& {\left[\begin{array}{c}
\mathbf{a}_{x y} \\
\beta_{z} \mathbf{a}_{x y} \\
\vdots \\
\beta_{z}^{N-1} \mathbf{a}_{x y}
\end{array}\right]} \\
& =\left[\begin{array}{c}
\mathbf{C}_{1} \mathbf{a}_{x y}+\mathbf{C}_{2} \beta_{z} \mathbf{a}_{x y} \\
\mathbf{C}_{2} \mathbf{a}_{x y}+\mathbf{C}_{1} \beta_{z} \mathbf{a}_{x y}+\mathbf{C}_{2} \beta_{z}^{2} \mathbf{a}_{x y} \\
\vdots \\
\mathbf{C}_{2} \beta_{z}^{N-3} \mathbf{a}_{x y}+\mathbf{C}_{1} \beta_{z}^{N-2} \mathbf{a}_{x y}+\mathbf{C}_{2} \beta_{z}^{N-1} \mathbf{a}_{x y} \\
\mathbf{C}_{2} \beta_{z}^{N-2} \mathbf{a}_{x y}+\mathbf{C}_{1} \beta_{z}^{N-1} \mathbf{a}_{x y}
\end{array}\right]
\end{aligned}
$$

Define the steering vector of the $n$th UCA as $\mathbf{a}_{n}=$ $\beta_{z}^{n-1} \mathbf{a}_{x y}, n=1, \ldots, N$. Since the submatrices $\mathbf{C}_{1}$ and $\mathbf{C}_{2}$ are Toeplitz matrices, the following equation can be derived [10].

$$
\mathbf{C}_{i} \mathbf{a}_{n}=\mathbf{T}_{n} \mathbf{c}_{i}, \quad i=1,2, n=1, \ldots, N
$$

in which $\mathbf{c}_{1}=\left[1, c_{x}\right]^{T}, \mathbf{c}_{2}=\left[c_{z}, c_{x z}\right]^{T}, \mathbf{T}_{n}=\mathbf{T}_{n 1}+\mathbf{T}_{n 2}+\mathbf{T}_{n 3}+$ $\mathbf{T}_{n 4} \mathbf{c}_{1}=\left[1, c_{x}\right]^{\mathrm{T}}$ is a matrix of size $M \times 2$ and

$$
\begin{aligned}
& {\left[\mathbf{T}_{n 1}\right]_{i, j}= \begin{cases}{\left[\mathbf{a}_{n}\right]_{i+j-1},} & i+j \leq M+1, \\
0, & \text { otherwise, }\end{cases} } \\
& {\left[\mathbf{T}_{n 2}\right]_{i, j}= \begin{cases}{\left[\mathbf{a}_{n}\right]_{i-j+1},} & i \geq j \geq 2, \\
0, & \text { otherwise, }\end{cases} } \\
& {\left[\mathbf{T}_{n 3}\right]_{i, j}= \begin{cases}{\left[\mathbf{a}_{n}\right]_{i-j+1+M},} & i<j \leq p, \\
0, & \text { otherwise, }\end{cases} } \\
& {\left[\mathbf{T}_{n 4}\right]_{i, j}= \begin{cases}{\left[\mathbf{a}_{n}\right]_{i+j-1-M},} & 2 \leq j \leq p, i+j \geq M+2, \\
0, & \text { otherwise, }\end{cases} }
\end{aligned}
$$

where $p=M / 2$ when $M$ is even and $p=(M+1) / 2$ when $M$ is the odd. Then (19) can be rewritten as

$$
\begin{aligned}
\mathbf{C a} & =\left[\begin{array}{c}
\mathbf{T}_{1} \mathbf{c}_{1}+\mathbf{T}_{2} \mathbf{c}_{2} \\
\mathbf{T}_{1} \mathbf{c}_{2}+\mathbf{T}_{2} \mathbf{c}_{1}+\mathbf{T}_{3} \mathbf{c}_{2} \\
\vdots \\
\mathbf{T}_{N-2} \mathbf{c}_{2}+\mathbf{T}_{N-1} \mathbf{c}_{1}+\mathbf{T}_{N} \mathbf{c}_{2} \\
\mathbf{T}_{N-1} \mathbf{c}_{2}+\mathbf{T}_{N} \mathbf{c}_{1}
\end{array}\right] \\
& =\left[\begin{array}{cc}
\mathbf{T}_{1} & \mathbf{T}_{2} \\
\mathbf{T}_{2} & \mathbf{T}_{1}+\mathbf{T}_{3} \\
\vdots & \vdots \\
\mathbf{T}_{N-1} & \mathbf{T}_{N-2}+\mathbf{T}_{N} \\
\mathbf{T}_{N} & \mathbf{T}_{N-1}
\end{array}\right] \cdot\left[\begin{array}{l}
\mathbf{c}_{1} \\
\mathbf{c}_{2}
\end{array}\right]=\mathbf{T} \mathbf{c}
\end{aligned}
$$

in which $\mathbf{c}=\left[1, c_{x}, c_{z}, c_{x z}\right]^{T}$ is the vector of mutual coupling coefficients. Substituting (22) into (18), we have

$$
\mathbf{U}_{N}^{H} \mathbf{T c}=\mathbf{0} .
$$

Define the matrix $\mathbf{Q}(\theta, \varphi)=\mathbf{T}^{H} \mathbf{U}_{N} \mathbf{U}_{N}^{H} \mathbf{T}$, and the estimation of the DOAs can be obtained by the $K$ roots of the following equation.

$$
\mathbf{c}^{H} \mathbf{Q}(\theta, \varphi) \mathbf{c}=0
$$

Because matrix $\mathbf{Q}(\theta, \varphi)$ is a Hermite matrix and $\mathbf{c} \neq \mathbf{0}$, (24) holds if and only if matrix $\mathbf{Q}(\theta, \varphi)$ is singular. Generally, matrix $\mathbf{Q}(\theta, \varphi)$ is full rank if $M N-K \geq 4$, while it will be singular when $(\theta, \varphi)$ coincides with one of the true signal directions $\left(\theta_{1}, \varphi_{1}\right), i=1, \ldots, K$. Therefore, the following azimuth estimators can be derived.

$$
\begin{array}{ll}
\widehat{\theta}_{i}=\underset{\theta}{\arg \max }\left\{\frac{1}{\operatorname{det}\left[\mathbf{Q}\left(\theta, \widehat{\varphi}_{i}\right)\right]}\right\}, & i=1, \ldots, K, \\
\widehat{\theta}_{i}=\underset{\theta}{\arg \max }\left\{\frac{1}{\lambda_{\min }\left[\mathbf{Q}\left(\theta, \widehat{\varphi}_{i}\right)\right]}\right\}, & i=1, \ldots, K,
\end{array}
$$

where $\operatorname{det}[\mathbf{Q}]$ is the determinant of matrix $\mathbf{Q}, \lambda_{\min }[\mathbf{Q}]$ is the smallest eigenvalue of $\mathbf{Q}$, and $\mathbf{v}_{\min }\{\mathbf{Q}\}$ is the corresponding eigenvector. It is noteworthy that each azimuth only needs a 1-D spectrum searching with the fixed elevation to get its estimation, which significantly reduces the calculation amount.

Based on the estimation of DOAs, the mutual coupling coefficient vector $\mathbf{c}$ can be obtained as follows.

$$
\mathbf{c}=\mathbf{v}_{\min }\{\mathbf{Q}(\widehat{\theta}, \widehat{\varphi})\}, \quad \text { with } \mathbf{v}_{\min }(1)=1
$$

The above derivation process takes advantage of the special structure of the array MCM, and the method developed here cannot be applied to arbitrary conformal arrays. In spite of that, it is still applicable to the arrays that 
TABLE 1: The CPU time of the proposed algorithm and the RARE $\operatorname{method}(\mathrm{s})$.

\begin{tabular}{lcc}
\hline Array size $(M \times N)$ & RARE & Proposed algorithm \\
\hline $16 \times 6$ & 1633.3164 & 0.7974 \\
$16 \times 8$ & 1861.5044 & 1.0987 \\
$16 \times 10$ & 2267.4448 & 1.4970 \\
\hline
\end{tabular}

have similar column structures, which are commonly used in practice.

\section{Simulation Results}

In this section, several simulations are carried out to illustrate the performance of the proposed method. An $M \times N$ uniformly spaced cylindrical array is considered in the following experiments. The observation range of azimuth and elevation of signals are both confined in $\left[0^{\circ}, 90^{\circ}\right]$, and the spectrum scanning step is $0.1^{\circ}$. The space between two neighbouring elements in a UCA is $d_{x y}=0.4 \lambda$, and the distance between two adjacent UCAs is $d_{z}=0.5 \lambda$. The two narrowband uncorrelated signals impinging on the array from far-field directions are $\left(\theta_{1}, \varphi_{1}\right)=\left(20^{\circ}, 30^{\circ}\right)$ and $\left(\theta_{2}, \varphi_{2}\right)=\left(40^{\circ}, 50^{\circ}\right)$. The mutual coupling coefficients are assumed to be $\mathbf{c}_{1}$ $[1,0.5+0.2 i]^{T}$ and $\mathbf{c}_{2}=[0.4+0.1 i, 0.22-0.14 i]^{T}$. Each element is assumed to have the following pattern in its local coordinate system.

$$
g\left(\theta^{\prime}, \varphi^{\prime}\right)= \begin{cases}{\left[1+\cos \left(\varphi^{\prime}\right)\right] / 2,} & 0 \leq \varphi^{\prime} \leq \pi / 2 \\ 0, & \text { otherwise }\end{cases}
$$

where $\theta^{\prime}$ and $\varphi^{\prime}$ are the azimuth and elevation in the local coordinate system for each element. Element pattern $g_{i}(\theta, \varphi)$ in the above analysis is a rotation of $g\left(\theta^{\prime}, \varphi^{\prime}\right)$.

For the DOA estimation method, the most timeconsuming process is the spectrum searching, especially in 2 -D condition. Under the prescribed condition, the proposed method needs to calculate $901 \times 2=1802$ points of the spatial spectrum, while 2-D RARE needs to calculate $901 \times 901=811,801$ points of the spatial spectrum. Although the amount of calculation will increase along with the number of signals, the proposed method still greatly reduces computational complexity.

In the first simulation, the computational complexity is analyzed by comparing the CPU time of the proposed algorithm and the RARE method. Comparisons are carried out for different sizes of arrays. Assume $S N R=10 \mathrm{~dB}$ and the number of snapshots is 500. With the Intel (R)Core (TM) i5 CPU $3.2 \mathrm{GHz}$ and 4GB RAM, the elapsed time of getting the DOA estimation results for both the proposed method and the RARE method is shown in Table 1. It is shown that the proposed method has a much smaller computing time than RARE and does not increase with the size of the array obviously.

In the second simulation, statistical experiments are carried out to analyze the estimation accuracy of the proposed method. 2-D RARE and MUSIC are also implemented

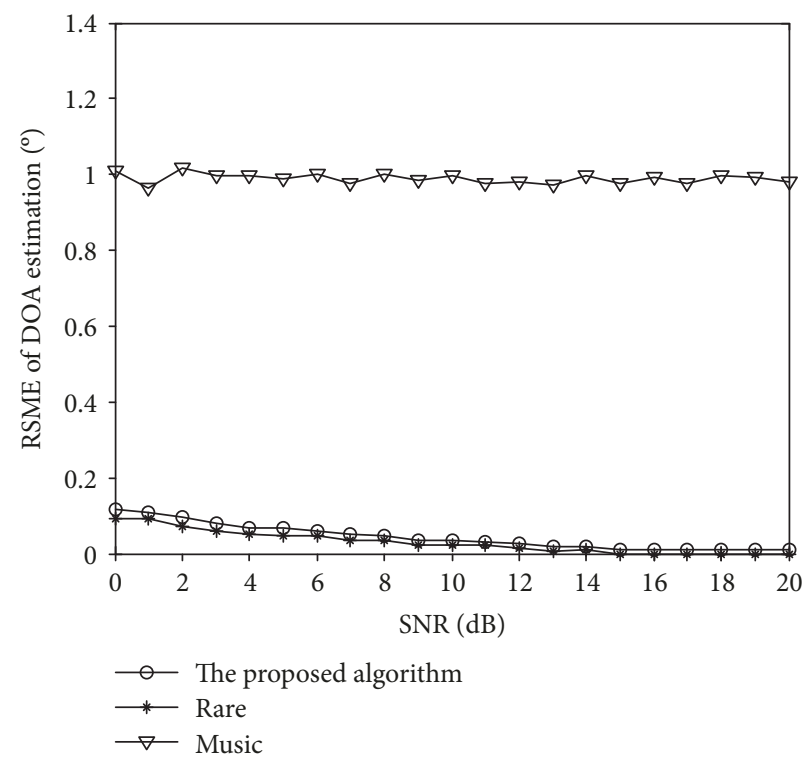

FIgURE 3: RMSE of DOA estimation versus SNR with 500 snapshots.

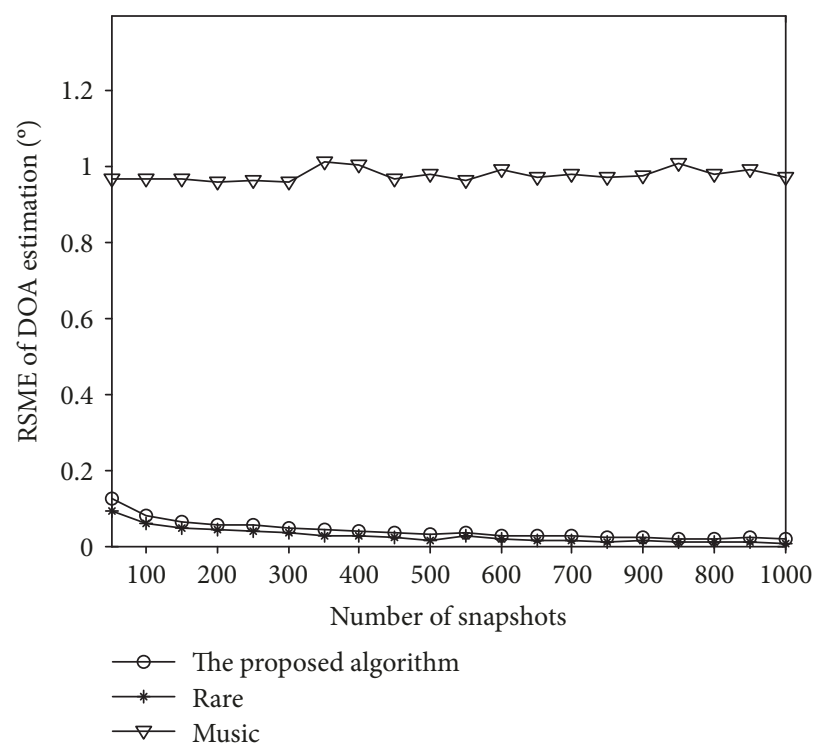

FIGURE 4: RMSE of DOA estimation versus the number of snapshots with $\mathrm{SNR}=10 \mathrm{~dB}$.

as comparison methods. A uniform CCA with the size of $16 \times 10$ is used here. 100 Monte Carlo trails are performed for each simulation and the DOA estimation accuracy is measured by the RMSE defined as follows.

$$
\operatorname{RMSE}_{\theta}=\sqrt{\frac{1}{100 K} \sum_{i=1}^{100} \sum_{k=1}^{K}\left\|\hat{\boldsymbol{\theta}}_{k}^{i}-\boldsymbol{\theta}_{k}\right\|^{2}},
$$

where $\widehat{\boldsymbol{\theta}}_{k}^{i}=\left(\widehat{\theta}_{k}^{i}, \widehat{\varphi}_{k}^{i}\right)$ is the estimation of $\boldsymbol{\theta}_{k}=\left(\theta_{k}, \varphi_{k}\right)$ in the $i$ th Monte Carlo experiment. Figure 3 shows the RMSE of the DOA estimation versus SNR with fixed 500 snapshots. Figure 4 shows the RMSE of the DOA estimation versus the number of snapshots with fixed $\mathrm{SNR}=10 \mathrm{~dB}$. These figures 


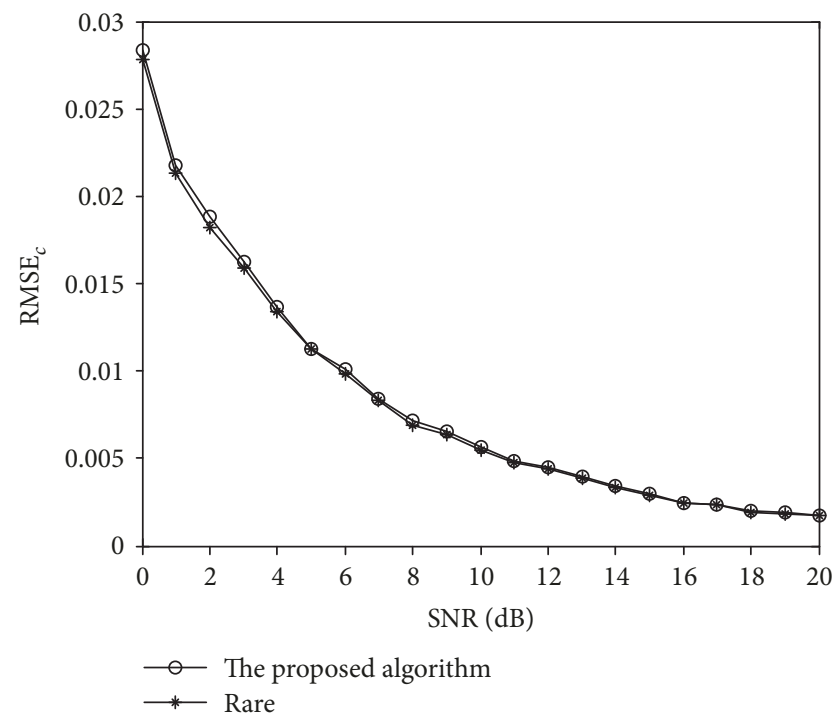

FIGURE 5: RMSE of the coupling coefficients versus SNR with 500 snapshots.

clearly indicate that the proposed algorithm can well compensate the mutual coupling effect and achieve satisfactory performance for the DOA estimation. The RMSE decreases with the increase of SNR and the number of snapshots as expected. Since there is a little aperture loss in the process of elevation estimation, the estimation accuracy of the proposed method is slightly lower than that of 2-D RARE. However, it is tolerable for this little performance loss with the distinct improvement in computational complexity.

Based on the estimation of DOAs, the mutual coupling coefficients can be obtained. To demonstrate its estimation accuracy, the RMSE of the mutual coupling coefficients is similarly defined as follows.

$$
\operatorname{RMSE}_{\theta}=\sqrt{\frac{1}{100 L} \sum_{i=1}^{100}\left\|\widehat{\mathbf{c}}_{i}-\mathbf{c}\right\|^{2}},
$$

where $\widehat{\mathbf{c}}_{i}$ is the estimation of $\mathbf{c}$ in the $i$ th Monte Carlo experiment and $L$ is the number of the mutual coupling coefficient.

The third simulation considers the same scenario as the second one. By using the above DOA estimation results, mutual coupling coefficients are subsequently estimated. Figure 5 shows the RMSE of the estimated coefficients versus the SNR with fixed 500 snapshots. Figure 6 shows the RMSE of the estimated coefficients versus the number of snapshots with fixed $S N R=10 \mathrm{~dB}$. Simulation results show that the estimation errors of mutual coupling coefficients for the two methods are very close. It can also be seen that higher estimation accuracy can be achieved with the larger SNR or the number of snapshots.

\section{Conclusion}

In this paper, an efficient 2-D DOA estimation method is proposed for the cylindrical conformal array in the presence of unknown mutual coupling. Through dimension reduction, the 2-D DOA estimation problem is divided into two 1-D

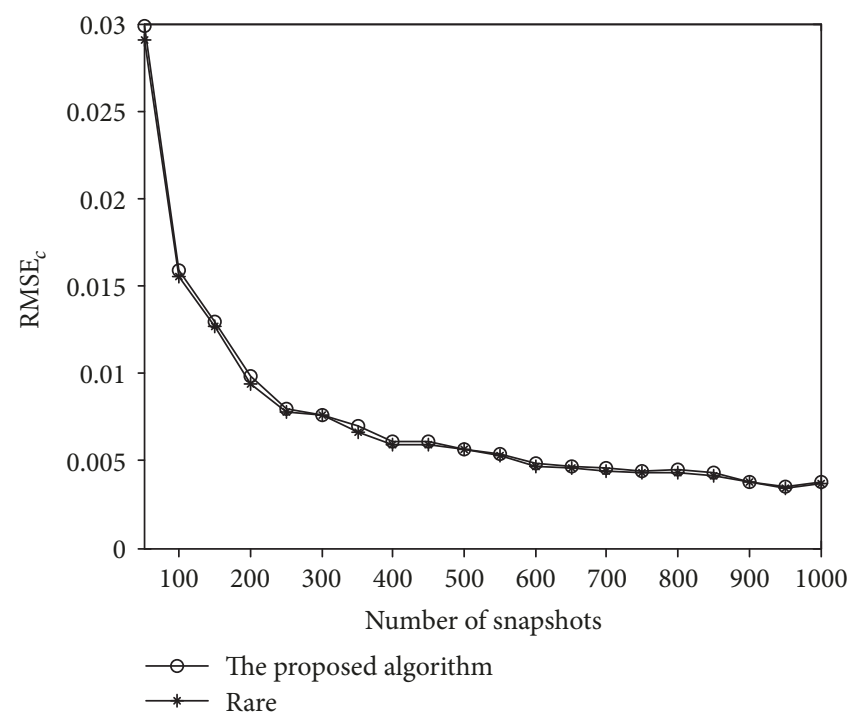

FIGURE 6: RMSE of the coupling coefficients versus the number of snapshots with $10 \mathrm{~dB}$.

DOA estimations for the cylindrical conformal array. The elevation and azimuth can be decoupled and estimated successively, and the mutual coupling coefficients can be obtained based on the DOA estimates. The proposed method avoids the complicated calculation of the 2-D spatial spectrum and can significantly reduce the amount of computation. Simulation results demonstrate the superior performance of the proposed method in terms of accuracy and computational efficiency.

\section{Data Availability}

The data used to support the findings of this study are available from the corresponding author upon request.

\section{Conflicts of Interest}

The authors declare that they have no conflicts of interest.

\section{Acknowledgments}

This work is supported by the Natural Science Foundation of Anhui Province of China (no. 1608085MF134).

\section{References}

[1] X. Lan, Y. Li, and Y. Chen, "A novel 2D DOA estimation via tensor modeling for cylindrical conformal array," in 2017 9th International Conference on Wireless Communications and Signal Processing (WCSP), pp. 1-6, Nanjing, China, October 2017.

[2] Y. Yu, H. Lui, C. Niow, and H. T. Hui, "Improved DOA estimations using the receiving mutual impedances for mutual coupling compensation: an experimental study," IEEE Transactions on Wireless Communications, vol. 10, no. 7, pp. 2228-2233, 2011. 
[3] A. Weiss and B. Friedlander, "Direction finding in the presence of mutual coupling," IEEE Transactions on Antennas and Propagation, vol. 39, no. 3, pp. 273-284, 1991.

[4] Z. Liu and Y. Zhou, "A unified framework and sparse Bayesian perspective for direction-of-arrival estimation in the presence of array imperfections," IEEE Transactions on Signal Processing, vol. 61, no. 15, pp. 3786-3798, 2013.

[5] Z. Ye and C. Liu, "On the resiliency of MUSIC direction finding against antenna sensor coupling," IEEE Transactions on Antennas and Propagation, vol. 56, no. 2, pp. 371-380, 2008.

[6] Z. Ye and C. Liu, "2-D DOA estimation in the presence of mutual coupling," IEEE Transactions on Antennas and Propagation, vol. 56, no. 10, pp. 3150-3158, 2008.

[7] Z. Ye, J. Dai, X. Xu, and X. Wu, "DOA estimation for uniform linear array with mutual coupling," IEEE Transactions on Aerospace and Electronic Systems, vol. 45, no. 1, pp. 280-288, 2009.

[8] Z. Ye, C. Liu, and Y. Zhang, "DOA estimation based on fourth-order cumulants with unknown mutual coupling," Signal Processing, vol. 89, no. 9, pp. 1839-1843, 2009.

[9] J. Dai and Z. Ye, "Spatial smoothing for DOA estimation of coherent signals in the presence of unknown mutual coupling," Signal Processing, vol. 5, no. 4, p. 418, 2011.

[10] C. Qi, Y. Wang, Y. Zhang, and H. Chen, "DOA estimation and self-calibration algorithm for uniform circular array," Electronics Letters, vol. 41, no. 20, pp. 1092-1094, 2005.

[11] B. Liao and S. Chan, "A cumulant-based method for direction finding in uniform linear arrays with mutual coupling," IEEE Antennas and Wireless Propagation Letters, vol. 13, pp. 1717-1720, 2014.

[12] B. Liao, Z. Zhang, and S. Chan, "DOA estimation and tracking of ULAs with mutual coupling," IEEE Transactions on Aerospace and Electronic Systems, vol. 48, no. 1, pp. 891-905, 2012.

[13] J. Dai, X. Bao, N. Hu, C. Chang, and W. Xu, "A recursive RARE algorithm for DOA estimation with unknown mutual coupling," IEEE Antennas and Wireless Propagation Letters, vol. 13, pp. 1593-1596, 2014.

[14] Q. Huang, H. Zhou, J. Bao, and X. Shi, "Mutual coupling calibration for microstrip antenna arrays via element pattern reconstruction method," IEEE Antennas and Wireless Propagation Letters, vol. 13, pp. 51-54, 2014.

[15] W. Mao, G. Li, X. Xie, and Q. Yu, "DOA estimation of coherent signals based on direct data domain under unknown mutual coupling," IEEE Antennas and Wireless Propagation Letters, vol. 13, pp. 1525-1528, 2014.

[16] K. Yang, Z. Zhao, W. Yang, and Z. Nie, "Direction of arrival estimation on cylindrical conformal array using RARE," Journal of Systems Engineering and Electronics, vol. 22, no. 5, pp. 767-772, 2011.

[17] X. Lan, L. Wang, Y. Wang, C. Choi, and D. Choi, “Tensor 2-D DOA estimation for a cylindrical conformal antenna array in a massive MIMO system under unknown mutual coupling," IEEE Access, vol. 6, pp. 7864-7871, 2018.

[18] T. Svantesson, "Modeling and estimation of mutual coupling in a uniform linear array of dipoles," in 1999 IEEE International Conference on Acoustics, Speech, and Signal Processing. Proceedings. ICASSP99 (Cat. No.99CH36258), vol. 5, pp. 2961-2964, Phoenix, AZ, USA, March 1999. 


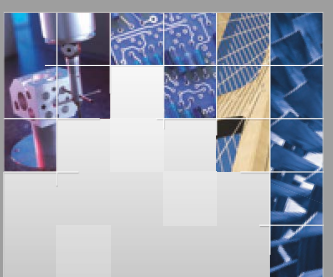

\section{Enfincering}
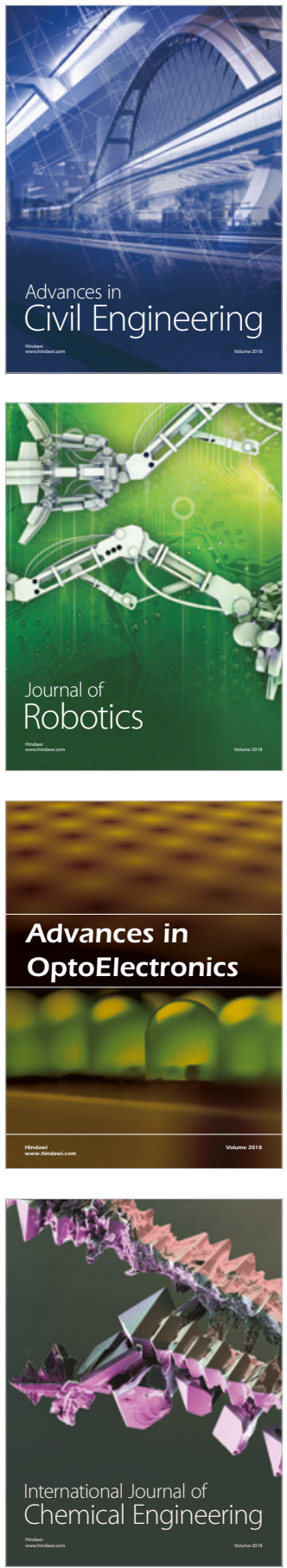

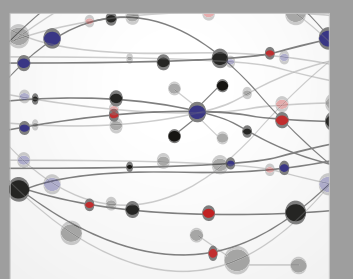

\section{Rotating \\ Machinery}

The Scientific World Journal

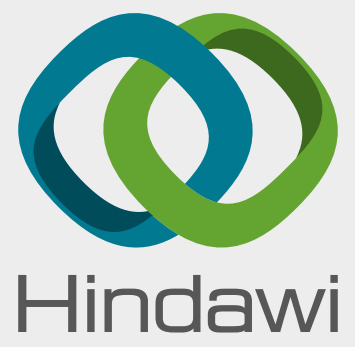

Submit your manuscripts at

www.hindawi.com
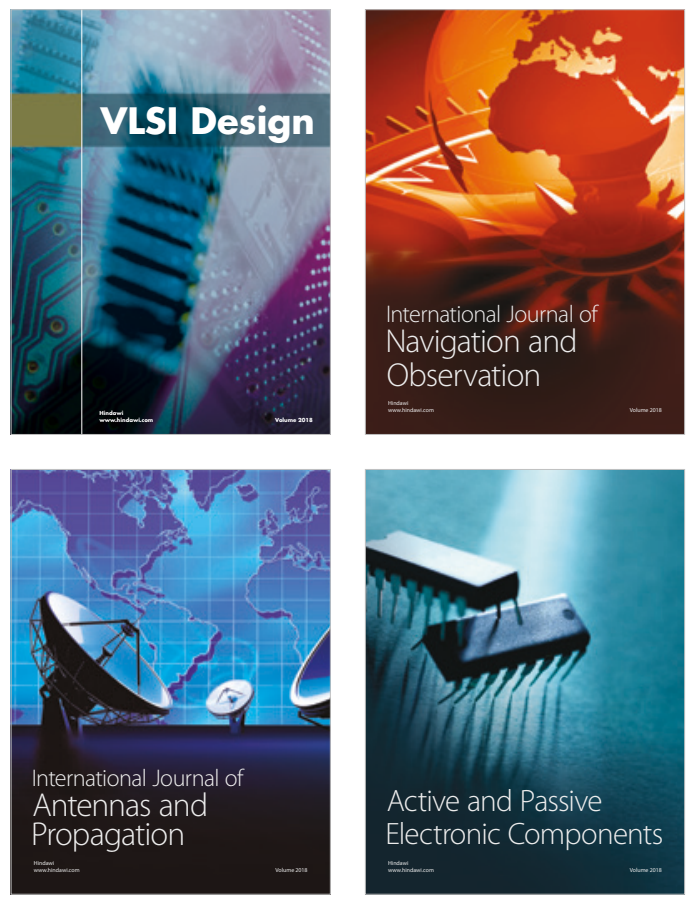
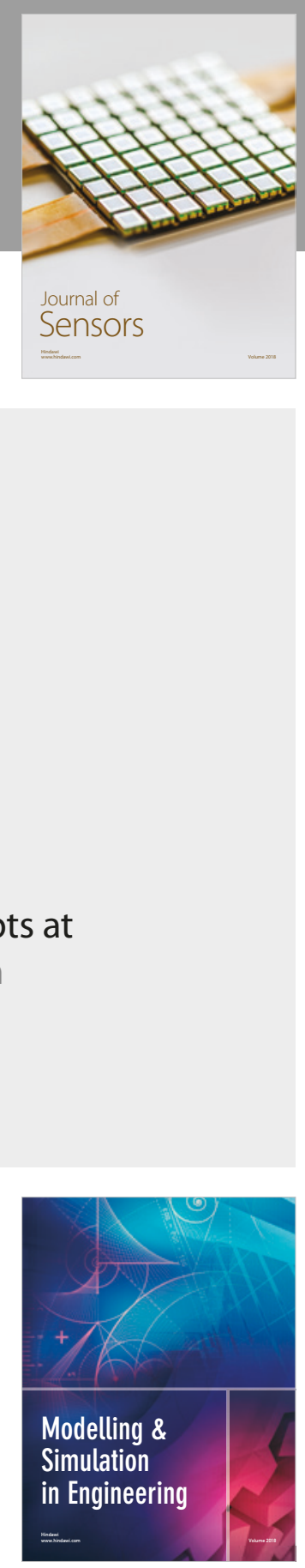

\section{Advances \\ Multimedia}
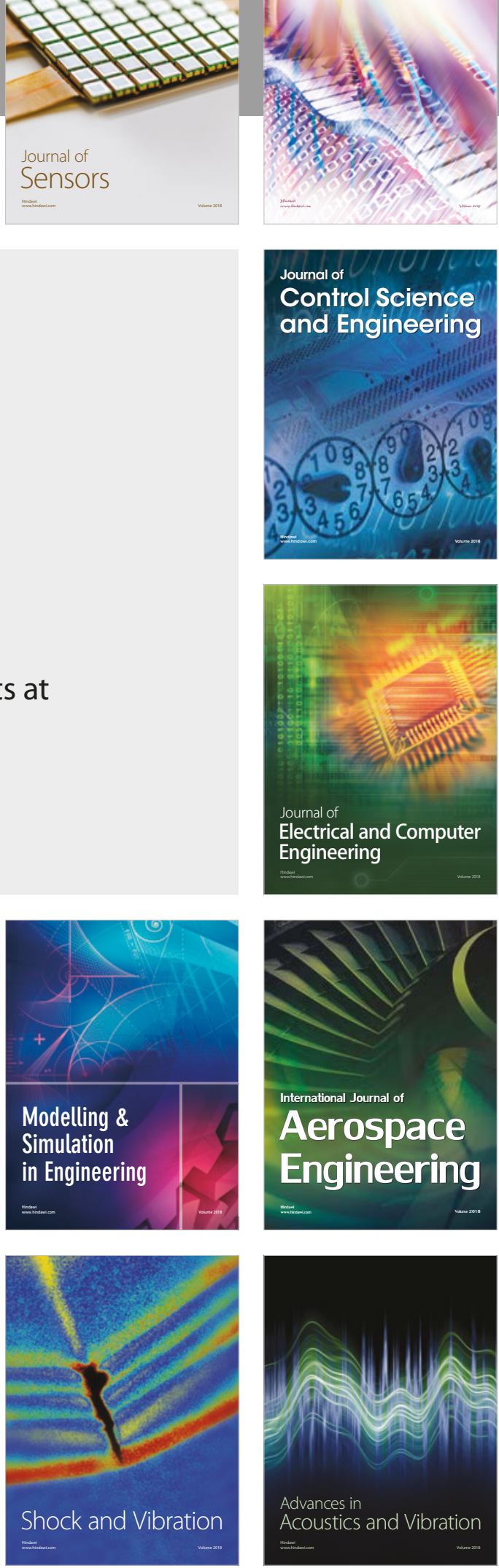